\title{
Testing the In-Vitro Product Performance of Nanomaterial-Based Drug Products: View of the USP Expert Panel
}

This stimuli article is the first in a series to be written by the USP Expert Panel on New Advancements in Product Performance Testing.

Matthias G Wacker ${ }^{1}$, Xujin Lu², Matt Burke ${ }^{3}$, Ishai Nir ${ }^{4}$, and Raafat Fahmy ${ }^{5}$

${ }^{1}$ Department of Pharmacy, Faculty of Science, National University of Singapore, Singapore.

${ }^{2}$ Bristol Myers Squibb, New Brunswick, New Jersey, USA.

${ }^{3}$ Radius Health, Inc., Cambridge, Massachusetts, USA.

${ }^{4}$ Distek, Inc., North Brunswick, New Jersey, USA.

${ }^{5}$ US Food and Drug Administration, Center for Veterinary Medicine, Office of New Animal Drug Evaluation, Maryland, USA

Correspondence should be addressed to: Kahkashan Zaidi, Senior Principal Scientist, United States Pharmacopeia, 12601 Twinbrook Parkway, Rockville, MD 20852-1790; email: kxz@usp.org.

\section{ABSTRACT}

Today, a wide variety of nanomaterial-based drug products enter the US market, creating the need for reliable standards and technologies to measure their performance in-vitro. A growing number of new performance assays are evaluated for testing the drug release from nanomaterials. On the one hand, they include real time separation methods such as dialysis, fiber optical systems, and flow-separation techniques. On the other hand, sample-and-separate methods such as centrifugation, filtration, and solid-phase extraction are commonly used. In our evaluation of the existing practices, we provide guidance in method development and validation of release assays. Also, we discuss requirements for standardization and documentation of release data. Furthermore, we highlight the knowledge gaps and challenges associated with drug release testing of nanomaterial-based drug products.

\section{INTRODUCTION}

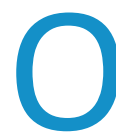

ver the years, a wide variety of nanomaterialbased drug products have entered the global healthcare market $(1,2)$. A recent USP chapter, Drug Products Containing Nanomaterials <1153>, provides clarification on terminology including liposomes, nanoparticles, nanocrystals, micelles, nanobubbles, nanofibers, nanotubes, nanoemulsions, and dendrimers. These novel dosage forms are characterized by exceptionally small dimensions and specific properties that make them more difficult to evaluate using conventional methodologies.

In the following article, recent trends and developments in Dissolution

Technologies FEBRUARY 2022

www.dissolutiontech.com the area of in-vitro performance testing of nanomaterialbased drug products will be discussed. For some of these dosage forms such as semisolids or inhalation products, the sample collection plays an important role. These issues will be discussed in more detail by other Stimuli articles and are beyond the scope of the present work.

\section{CURRENT USP FRAMEWORK}

Currently, <1153> summarizes dosage forms that exhibit specific features related to the use of nanotechnology and provides the terminology to be used in the context of the USP framework. To a certain extent, the versatility of drug delivery concepts is reflected by the performance parameters that have been discussed for 
nanomaterials. These performance parameters include the drug release, the physical stability or "dispersibility" of colloids, the ability of the carrier to protect the drug from degradation, as well as the release of compounds into specific compartments known to influence the biodistribution, such as the plasma proteins (3) or lipids (4). This versatility is reflected in different sections of the USP as well. For example, Gene Therapy Products <1047> recognizes the use of liposomes or lipid complexes to enhance cell penetration of deoxyribonucleic acid (DNA) molecules as well as the impact of complexation on the shelf life of the drug product. Other than the dissolution of the drug, the protective effect of the material on the compound essentially contributes to the performance of the drug product.

Conversely, In Vitro Release Test Methods for Parenteral Drug Preparations <1001> emphasizes the drug release and provides selected information on some of the most common methods applied to the testing of nanosuspensions and liposomes.

In the future, the interplay between the release and stabilization of drugs will play a more dominant role. Therefore, the next generation of release media should simulate both, the microenvironment relevant for dissolution and release of the drug as well as the multiple influences on drug and formulation stability (5). In this article, we focus on separation and isolation methods used in the detection of the free and the particlebound fractions of the drug. Also, we provide some recommendations for the development and validation of the release assay.

\section{REAL-TIME SEPARATION METHODS}

Real-time separation methods apply continuous separation or detection to identify the free fraction and the encapsulated fraction of the drug. In the following sections, these methods will be discussed in more detail. Selected assays and protocols described in the current literature are provided in Table 1 . They follow certain standards but represent only a small fraction of those available in the literature.

\section{Dialysis Methods Background}

Today, a variety of dialysis methods are considered to measure the drug release from nanomaterial-based drug products. They utilize the inherent barrier properties of the dialysis membrane to separate colloids from drug molecules and proteins (Fig. 1). Commonly, the dosage form is filled into the donor chamber. The donor compartment is separated from the acceptor compartment by a dialysis membrane. After injection of the sample into the donor chamber, the concentration gradient between both compartments drives the exchange of molecules. The free drug is quantified from the acceptor compartment. Reverse dialysis refers to a process where a compartment that is larger in volume is used as the donor compartment.

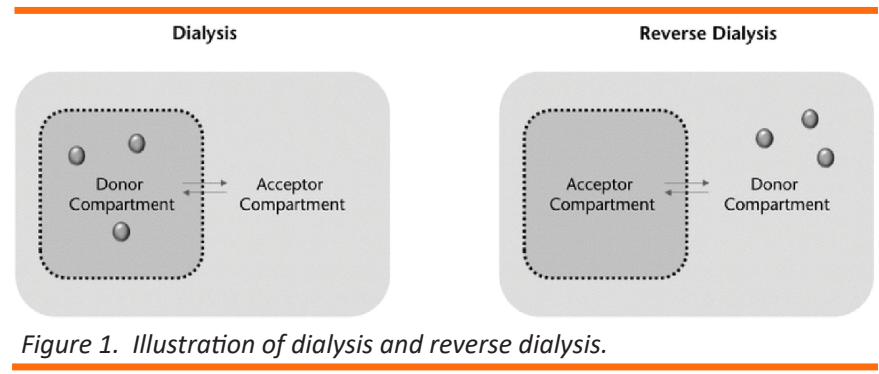

For both setups, there are two kinetic processes involved in the drug release profile: the release of the drug from the carrier and the permeation of the drug through the dialysis membrane. The rate of the membrane flux $(\mathrm{J})$ is described by Fick's law of diffusion and depends on the diffusion coefficient $(D)$, the membrane surface area $(A)$, the concentration gradient between the donor and the acceptor compartment $(d c)$, and the thickness of the diffusion layer ( $h$ ).

$$
\mathrm{J}=(D \times A \times d c) / h
$$

It can be summarized in the membrane permeation rate constant $(k M)$. The medium exchange between both compartments is affected by membrane permeability as well (18). Conventional approaches often use dialysis cassettes or tubes with a well-defined surface area (19). They come with several technical challenges such as the agglomeration of the nanomaterial in the donor chamber. This can lead to the formation of a diffusion layer and, in consequence, to prolonged membrane transport. In this context, special attention should be paid to the viscosity of the drug product in the donor and the acceptor chambers (20). For validation purposes, the permeability of the dialysis membrane should be tested before and after the release experiment with a solution of the drug to make sure that there is no significant delay in the release. A slow membrane transport may also lead to a violation of sink conditions because of a temporary saturation with drug molecules inside the donor chamber (18). Therefore, the release response for dialysis processes expressed as the membrane permeation rate constant should be determined $(3,21)$. The significance of the separation time for the sensitivity of the method widely depends on the performance characteristics of the product. Therefore, to avoid the risk of undetected batch-to-batch differences,

Dissolution'

FEBRUARY 2022 Technologies www.dissolutiontech.com 
Table 1. Descriptions of Selected Methodologies Applied in the Separation of Nanomaterial-Based Drug Products

\begin{tabular}{|c|c|c|c|c|c|c|c|c|c|}
\hline $\begin{array}{c}\text { Nanomaterial } \\
<1153>\end{array}$ & $\begin{array}{l}\text { Intended } \\
\text { Admin } \\
\text { Route }\end{array}$ & Description & Apparatus & $\begin{array}{l}\text { Separation } \\
\text { Method }\end{array}$ & $\begin{array}{l}\text { Analytical } \\
\text { Methods }\end{array}$ & $\begin{array}{l}\text { Additional } \\
\text { Information }\end{array}$ & Medium & $\begin{array}{l}\text { Applica- } \\
\text { tion }\end{array}$ & Ref \\
\hline Dendrimers & IV & $\begin{array}{l}\text { Thermoshaker with } \\
\text { sample collection }\end{array}$ & $\begin{array}{l}\text { Noncom } \\
\text { pendial }\end{array}$ & Dialysis & UV & $\begin{array}{c}\text { Amphotericin } \\
\text { B-loaded } \\
\text { polyamidoamine } \\
\text { (PAMAM) dendrimers }\end{array}$ & PBS & $\mathrm{BR}$ & (6) \\
\hline $\begin{array}{c}\text { Drug } \\
\text { nanoparticles } \\
\text { or nanocrystals }\end{array}$ & IV & $\begin{array}{l}\text { Apparatus } 1 \text { and } \\
2 \text { with dispersion } \\
\text { releaser }\end{array}$ & $\begin{array}{l}\text { USP } 1 \\
\text { and } 2\end{array}$ & Dialysis & $\begin{array}{l}\text { HPLC + } \\
\text { NTA }\end{array}$ & $\begin{array}{l}\text { Temoporfin } \\
\text { nanocrystals }\end{array}$ & $\begin{array}{c}\text { PBS }+10 \% \\
\text { serum }+ \\
\text { cyclodextrin }\end{array}$ & $\mathrm{BR}$ & (7) \\
\hline $\begin{array}{c}\text { Drug } \\
\text { nanoparticles } \\
\text { or nanocrystals }\end{array}$ & $\mathrm{PO}$ & $\begin{array}{l}\text { Standard dissolution } \\
\text { test with syringe } \\
\text { filter separation }\end{array}$ & USP2 & $\begin{array}{l}\text { Syringe } \\
\text { filtration }\end{array}$ & $\begin{array}{l}\text { HPLC + } \\
\text { NTA }\end{array}$ & $\begin{array}{c}\text { Nanocrystals } \\
\text { comprising } \\
\text { fenofibrate }{ }^{a} \text { and other } \\
\text { formulations }\end{array}$ & $\begin{array}{l}\text { FaSSGF, } \\
\text { FaSSIF }\end{array}$ & $\mathrm{BR}$ & (8) \\
\hline Liposomes & IV & $\begin{array}{l}\text { Apparatus } 1 \text { and } \\
2 \text { with dispersion } \\
\text { releaser }^{b}\end{array}$ & $\begin{array}{l}\text { USP } 1 \\
\text { and } 2\end{array}$ & Dialysis & $\begin{array}{l}\text { HPLC + } \\
\text { NTA }\end{array}$ & $\begin{array}{l}\text { Temoporfin- loaded } \\
\text { non- PEGylated and } \\
\text { PEGylated liposomes }{ }^{c}\end{array}$ & $\begin{array}{c}\text { PBS+ } 10 \% \\
\text { serum + } \\
\text { cyclodextrins }\end{array}$ & $\mathrm{BR}$ & (9) \\
\hline $\begin{array}{l}\text { Liposomes or } \\
\text { nanocrystals }\end{array}$ & IV & $\begin{array}{l}\text { Apparatus } 1 \text { and } \\
2 \text { with dispersion } \\
\text { releaser }^{b}\end{array}$ & $\begin{array}{l}\text { USP } 1 \\
\text { and } 2\end{array}$ & Dialysis & HPLC & $\begin{array}{c}\text { Temoporfin in } \\
\text { organic solution and } \\
\text { temoporfin- loaded } \\
\text { liposomes }^{d}\end{array}$ & $\begin{array}{c}\text { PBS+ 0\%- } \\
50 \% \text { serum + } \\
\text { cyclodextrins }\end{array}$ & $\mathrm{BR}$ & (3) \\
\hline Liposomes & IV & $\begin{array}{c}\text { Apparatus } 4 \text { with } \\
\text { dialysis sac with } \\
\text { holder }^{\text {e }}\end{array}$ & USP4 & Dialysis & HPLC & Amphotericin $B^{f}$ & $\begin{array}{c}\text { HEPES + } \\
\text { sucrose + } \\
\text { cyclodextrins }\end{array}$ & QC & (10) \\
\hline Liposomes & IV & $\begin{array}{l}\text { Apparatus } 4 \text { with } \\
\text { adapter for dialysis }\end{array}$ & USP4 & Dialysis & HPLC & $\begin{array}{l}\text { Dexamethasone- } \\
\text { loaded liposomes }\end{array}$ & HEPES + SDS & QC & (11) \\
\hline Micelles & IV & $\begin{array}{l}\text { Apparatus } 2 \text { with } \\
\text { dialysis bag }\end{array}$ & USP2 & Dialysis & $\begin{array}{l}\text { HPLC + } \\
\text { DLS }\end{array}$ & $\begin{array}{l}\text { Dexamethasone- } \\
\text { loaded liposomes }\end{array}$ & PBS & QC & (12) \\
\hline Nanobubbles & IV & $\begin{array}{c}\text { Dialysis bag in a } \\
\text { noncompendial } \\
\text { beaker }\end{array}$ & $\begin{array}{l}\text { Noncom } \\
\text { pendial }\end{array}$ & Dialysis & HPLC & $\begin{array}{l}\text { Doxorubicin- loaded } \\
\text { nanobubble }\end{array}$ & PBS & $\mathrm{BR}$ & (13) \\
\hline Nanoemulsions & $\mathrm{PO}$ & $\begin{array}{l}\text { Apparatus } 2 \text { with } \\
\text { dialysis bag }\end{array}$ & USP2 & Dialysis & UV & $\begin{array}{l}\text { Nanoemulsions of } \\
\text { candesartan cilexetil }\end{array}$ & $\begin{array}{c}0.1 \mathrm{~N} \\
\text { hydrochloric } \\
\text { acid }\end{array}$ & QC & (14) \\
\hline Nanoemulsions & $\begin{array}{l}\text { Ocular } \\
\text { or IV }\end{array}$ & $\begin{array}{c}\text { Adaptive perfusion } \\
\text { system }\end{array}$ & USP2 & $\begin{array}{l}\text { Tangential } \\
\text { flow } \\
\text { filtration }\end{array}$ & HPLC & $\begin{array}{l}\text { Nanoemulsions of } \\
\text { difluprednate }\end{array}$ & $\begin{array}{l}\text { Phosphate } \\
\text { buffer (pH } \\
7.4)\end{array}$ & QC & (15) \\
\hline Liposomes & - & $\begin{array}{l}\text { Thermoshaker } \\
\text { setup with sample } \\
\text { collection }\end{array}$ & USP2 & $\begin{array}{l}\text { Ultrafiltrati } \\
\text { on and } \\
\text { dynamic } \\
\text { dialysis }\end{array}$ & HPLC & $\begin{array}{l}\text { Topotecan loaded } \\
\text { liposomes }\end{array}$ & $\begin{array}{l}\text { Formate } \\
\text { buffer }\end{array}$ & QC & (16) \\
\hline $\begin{array}{c}\text { Drug } \\
\text { nanoparticles }\end{array}$ & IV & $\begin{array}{l}\text { Thermoshaker } \\
\text { setup with sample } \\
\text { collection }\end{array}$ & $\begin{array}{l}\text { Noncom } \\
\text { pendial }\end{array}$ & $\begin{array}{l}\text { Ultracentri } \\
\text { fugation }\end{array}$ & HPLC & $\begin{array}{l}\text { Topotecan loaded } \\
\text { liposomes }\end{array}$ & $\begin{array}{l}\text { Plasma and } \\
\text { water with } \\
\text { polysorbate } \\
80\end{array}$ & QC & (17) \\
\hline Liposomes & Inhalation & Shaking water bath & $\begin{array}{l}\text { Noncom } \\
\text { pendial }\end{array}$ & $\begin{array}{l}\text { Centrifugal } \\
\text { filtration }\end{array}$ & HPLC & $\begin{array}{l}\text { Ciprofloxacin-loaded } \\
\text { liposomes }\end{array}$ & $50 \%$ serum & $\mathrm{BR}$ & (18) \\
\hline
\end{tabular}

${ }^{a}$ Lipidil; ${ }^{b}$ Pharma Test Apparatebau AG; ${ }^{c}$ Foslip and Fospeg; ${ }^{d}$ Foscan and Foslip; ${ }^{e}$ Float-A-Lyzer; ${ }^{f}$ Ambisome

$I V=$ intravenous; $P O=$ peroral; UV = ultraviolet spectroscopy; HPLC = high-performance liquid chromatography; NTA = nanoparticle tracking analysis; OLS = dynamic light scattering; PBS = phosphate buffered saline; FaSSGF = fasted state simulated gastric fluid; FaSSIF = fasted state simulated intestinal fluid; HEPES = 4-(2-hydroxyethyl)-1-piperazineethanesulfonic acid; SOS = sodium dodecyl sulfate; BR = biorelevant; $Q C=$ quality control. 
every dosage form should be evaluated individually. Reference experiments should be carried out to make sure that the dialysis-based separation has only minor influence on the performance measured by the assay.

To avoid strong interactions of the concentrated formulation with the membrane (22), reverse dialysis can be considered (19). While this method reduces particle interactions, a disadvantage is that it decreases the concentration gradient between the acceptor and the donor chamber. Consequently, there is a balance to be maintained between the volume required for a stable dispersion and the membrane flux.

\section{Method Development}

There are a wide variety of membranes available on the market with the molecular-weight cutoff (MWCO) ranging from 1-1000 kilodaltons $(\mathrm{kDa})$. The MWCO is the membrane size where approximately $90 \%$ of a solute with the respective molecular weight is retained by the membrane. These specifications are based on the measurement of membrane permeation using marker molecules such as bovine serum albumin or insulin. They do not represent a theoretical cutoff based on size but are the result of an actual separation problem. However, the MWCO of membrane materials may slightly change over time.
Manufacturers suggest the selection of an MWCO of $80 \%$ $90 \%$ more than the solute (23). A kinetic measurement depends on the surface area available for diffusion. Therefore, to maximize the membrane flux, the largest pore size still retaining approximately $95 \%$ of the particles should be selected. Quantification of the retention can be performed using well-defined particle standards (24). However, most of the commercial fluorescent particle standards release a certain fraction of the label after a few hours. So far there are no suitable standards with high stability over more than $24 \mathrm{~h}$.

In addition to the membrane pore size, the membrane material plays a critical role. Xie et al. compared the permeation rate under similar conditions including drug, medium, temperature, and pore size (25). The membrane material still had a strong impact on the permeation rate of the solute (25). The most common membrane materials are cellulose ester and regenerated cellulose. In a conventional dialysis experiment, because of the absence of competing shear forces in the donor chamber, even weak interactions with the dialysis membrane may lead to a significant delay in membrane permeation. Key parameters to be considered in the development of dialysis-based methods are summarized in Table 2 .

\section{Table 2. Key Parameters to be Explored and Documented for the Evaluation of Dialysis-Based Release Experiments}

\begin{tabular}{|c|c|}
\hline Molecular weight cutoff & $\begin{array}{l}\text { The molecular weight cutoff depends on the pore size of the membrane and determines the exchange of } \\
\text { molecules between the donor and acceptor compartments. The molecular weight cutoff is quantified by the } \\
\text { separation of marker molecules and the most relevant specification provided by the manufacturer. It is closely } \\
\text { related to the pore size of the membrane. }\end{array}$ \\
\hline $\begin{array}{l}\text { Surface area and thickness of the } \\
\text { membrane }\end{array}$ & $\begin{array}{l}\text { The porosity and membrane dimensions (i.e., surface area and thickness) affect the rate of membrane transport. } \\
\text { The thickness of the dialysis membrane is provided by the manufacturer. These parameters should be included in } \\
\text { the method description, particularly for those setups that do not follow well-defined standards and dimensions. }\end{array}$ \\
\hline $\begin{array}{l}\text { Volumes of donor and acceptor } \\
\text { compartments }\end{array}$ & $\begin{array}{l}\text { The drug concentration in the donor and the acceptor compartment is strongly affected by the concentration } \\
\text { gradient. The volume in the donor and acceptor chambers enables a calculation of the surface-to-volume ratio, } \\
\text { which represents an important performance characteristic of dialysis methods. }\end{array}$ \\
\hline Sample injection protocol & $\begin{array}{l}\text { Sample injection temporarily changes the composition of the medium, particularly for those methods that do } \\
\text { not involve agitation of the donor chamber. The sample injection protocol includes the exact composition of the } \\
\text { injected sample, injection rate, volume, and drug content. }\end{array}$ \\
\hline Material interaction & $\begin{array}{l}\text { Membrane adsorption of the drug should be investigated before the selection of a dialysis membrane. In this } \\
\text { context, exact hydrodynamics play an important role. It is therefore recommended to carry out the material } \\
\text { interaction studies under conditions very similar to the release test. Because of the difference in hydrodynamics, } \\
\text { an assay using a similar material but a different extraction method may not be suitable to predict the conditions in } \\
\text { the release assay. }\end{array}$ \\
\hline $\begin{array}{l}\text { Membrane cleaning or } \\
\text { conditioning method }\end{array}$ & $\begin{array}{l}\text { The membrane cleaning or conditioning method can be critical to the reproducibility of release experiments. } \\
\text { Depending on the membrane chemistry, additives are added to prevent damage to the membrane. This can } \\
\text { adversely impact the diffusion rate across the membrane and care should be taken to mitigate this influence. }\end{array}$ \\
\hline Release response & $\begin{array}{l}\text { The release response provides a time-resolved measure of the amount of free drug being detected when using } \\
\text { a specific separation process. Commonly, a solution of the free drug is spiked into the donor chamber, and the } \\
\text { amount of the drug that becomes available (the response) is measured in a time resolved manner. Importantly, } \\
\text { the time between the addition of free (solubilized drug) and the detection in the assay is reported. As compared to } \\
\text { a conventional spiking recovery test, the separation time is not considered negligible. }\end{array}$ \\
\hline
\end{tabular}




\section{Dialysis Bag Method}

The dialysis bag method is a release test, separating the released fraction from the particle-bound fraction of the drug. It uses dialysis tubes or cassettes as the donor ("normal" dialysis) or acceptor chamber ("reverse" dialysis). The setup has been described in combination with several instruments including but not limited to simple beakers and dissolution apparatuses 2 and 4 . For dissolution apparatus 4, some studies recommend the use of a holder cell or single-use dialysis devices (10).

The instrument commonly affects the hydrodynamics in the acceptor compartment considerably, while drug transport in the donor compartment is widely driven by diffusion of the compound through a resting diffusion layer.

The surface-to-volume ratio, the membrane pore size, the release medium (in the acceptor and the donor chamber), the localization of the dialysis bag in the acceptor compartment as well as the exact configuration of the dissolution apparatus (e.g., stirring rate, flow rate) can influence the outcome. The "dialysis bag" setup suggested for dissolution apparatus 4 uses an optimized surface-to-volume ratio and an optimized position in the center of the dissolution cell. Validation of the in-vitro release test was carried out with liposomal amphotericin B (Ambisome) (10).

\section{Dispersion Releaser}

The dispersion releaser (DR) (Pharma Test Apparatebau $A G)$ is a device for testing the drug release from nanomaterial-based drug products using a dissolution apparatus 2.

The system is connected to the shaft of the dissolution tester. A donor cell holds the dialysis membrane in the center position of the dissolution vessel (including standard vessel and mini-vessel configuration). The donor and the acceptor chamber are constantly agitated the same rate (Fig. 2). The formulation is injected into the donor chamber using a sampling port that can be used for sample injection and sample collection from the donor chamber. Due to the limited volume of the inner chamber, only a certain dose range can be injected into the donor compartment (depending on the drug load of the dispersed dosage form). So far, the setup has been evaluated for testing the drug release from nanoparticles $(21,26,27)$, liposomes $(3,9)$, nanocrystals $(7)$, and polymer micelles, using buffers (21) and biorelevant dissolution media $(3,7,9,26)$.

\section{Fiber Optical Systems Background}

Another approach to measuring the dissolution rate of nanoparticle formulations is by in-situ optical spectroscopic measurements performed directly in the dissolution apparatus, typically accomplished using fiber optical systems. Shifting the measurement in-situ enables real-time determination of the concentration of drugs. Since there is no delay between sampling and analysis, analytical errors due to the separation time are eliminated. As is also the case with in-situ measurements of conventional products, the drug concentration can be detected continuously, and the time resolution is considerably increased (25). This enables the collection of complete release profiles, even from the most rapidly dissolving drug products.

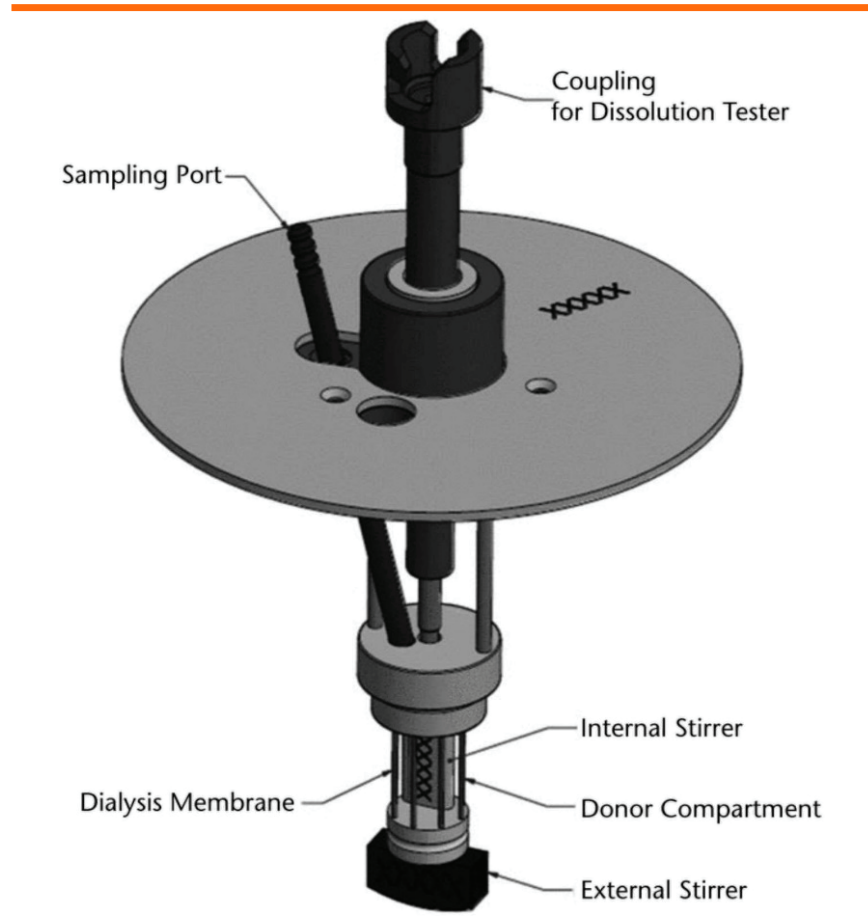

Figure 2. Schematic of the dispersion releaser. (Image adapted courtesy of Pharma Test Apparatebau AG).

While there is no separation protocol involved, the limitations of fiber optical systems arise from the background signal of all particles present in the formulation as well as from the components of the release medium. Nanomaterial-based products are more difficult to measure because the particles underlie Brownian motion and often remain well-dispersed for the whole duration of the release experiment. Noteworthy, not only the size but also the density of the particles affects their sedimentation behavior. Therefore, a detailed understanding of the optical properties and sedimentation behavior is required. For larger particles and excipients, the sedimentation kinetics sometimes 
changes in the composition of the medium, the particle size, and the quality of excipients may have a stronger impact on the outcome compared to other methods. Therefore, such changes should be accompanied by a partial revalidation.

\section{Method Development}

As outlined previously, undissolved particles, excipients, carriers, complex media, and other components may contribute to the background signal measurements. Since conventional filtering is generally not an option with in-situ optical measurements, mathematical data analyses have been developed to reduce the influence of these potential error sources. Many of the corrections are independent of particle size, allowing them to also be applied to many nanoformulations. Still, in some cases, these interferences cannot be reproducibly removed. See Table 3 for selected key parameters to be optimized in the development of fiber optical systems.

\section{Flow-Separation Methods Background}

In recent years, an increasing number of flow-separation techniques have been applied to measure the drug release from nanomaterial formulations. Among other approaches, asymmetric flow field-flow fractionation (AF4) was used to isolate the free and the protein-bound drug (28). In literature, the methodology has been applied to investigate the drug release from liposomes (29) and drug conjugates (28). While drug conjugates are defined by their chemical structure, most nanomedicines described in USP are multimolecular assemblies with a physicochemical rather than a unique chemical identity. For these formulations, a strong impact of the separation time, the dilution with the carrier liquid, and the shear forces applied during separation play an eminent role.

Holzschuh et al. diluted samples with carrier liquid followed by a separation time of $60 \mathrm{~min}$ in the AF4 without further inhibition of the drug release (29). The protein partitioning was measured after the particles passed the flow channel without further exploring the impact of the separation method on the release behavior. Caputo et al. reported separation times of 40-80 $\mathrm{min}$ for the investigation of drug partitioning (28). Although the method was suitable to measure the release from examples of drug conjugates (28) and slowreleasing liposomes (30), this methodology may not be suitable for other drug formulations. This highlights the need for guidance in the identification of suitable separation methods and method validation. So far, some of the conclusions proclaiming AF4 as "one of the chosen techniques for quality control" (28) are not based on hard evidence and widely ignore the limitations outlined by other authors (31).

Table 3. Key Parameters to be Explored and Documented for the Evaluation of Release Experiments Using Fiber Optical Methods

\begin{tabular}{|c|c|}
\hline Optical method & $\begin{array}{l}\text { Focusing on absorbance (typically performed in the ultraviolet region), both instrument design and measurement } \\
\text { parameters contribute to the accuracy, precision, and robustness of the analysis. Thus, a description of the method } \\
\text { should include the design of the optical system. Standard spectrophotometer options include: } \\
\text { Multiplexed scanning system } \\
\text { Multiplexed single multichannel (diode array type) systems } \\
\text { Multiple independent multichannel (diode array type) systems } \\
\text { Single multichannel (2-dimensional type) system }\end{array}$ \\
\hline Probe type and location & $\begin{array}{l}\text { The design and location of the optical probes can affect the hydrodynamics and the vulnerability of the probe to air } \\
\text { bubbles or particles present in the media transiting the path length. Standard probe options include: } \\
\text { Transflectance (also known as dip) probes } \\
\text { Arch probes }\end{array}$ \\
\hline Spectral analysis parameters & $\begin{array}{l}\text { The choice of the spectral analysis parameters used in calculating the dissolution rate may need optimization or } \\
\text { qualification. Options include: } \\
\text { Measurement wavelength or wavelength range } \\
\text { Analytical wavelength or summed wavelength range } \\
\text { Blank measurements: Are they performed in dissolution media? Do they include a capsule blank?, etc. } \\
\text { Standards measurements: Corresponding to what percent dissolved? Does the media differ from the dissolution media? }\end{array}$ \\
\hline Data processing & $\begin{array}{l}\text { Data treatment sometimes goes beyond measuring simple peak values and may include: } \\
\text { Algorithms for baseline correction (e.g., offset correction, the slope between two points) } \\
\text { Derivative spectroscopy (e.g., first or second derivative, single point, or range analysis) } \\
\text { More-complex spectral modeling (e.g., scattering modeling, multicomponent analysis, other chemometric techniques). } \\
\text { These algorithms must be reported for interpretation of the release profile. }\end{array}$ \\
\hline $\begin{array}{l}\text { Hydrodynamics and } \\
\text { dispersibility }\end{array}$ & $\begin{array}{l}\text { Fluctuations in the background signal are an important error source. For example, the influence of hydrodynamics, } \\
\text { agglomeration, and sedimentation behavior should be monitored, as these may require changes in the data correction } \\
\text { algorithm. Although these algorithms are designed to cover a wide range of dosage forms, orthogonal methods should } \\
\text { be used during method validation. Further, considerable changes in excipient quality, particle size, or the conditions } \\
\text { used by the performance test may require partial revalidation of the method. }\end{array}$ \\
\hline
\end{tabular}


Other flow-separation methods include the NanoDis (Agilent Technologist, USA). It uses an automated crossflow filtration for the separation of particle populations from the release medium. So far, the drug release has been tested in buffer media. As compared to AF4, the separation time has been reduced significantly. Because the NanoDiS is an open filtration system, the risk of a filter cake formation is reduced considerably. Still, the efficiency and the time required to collect the filtrate depend on the exact dosage form and the concentration of nanoparticles in the medium. The NanoDis system operates in filtration mode to support the sampling of the free drug. It removes small amounts from the release apparatus (e.g., USP apparatus 2). Therefore, it has only a minor influence on the microenvironment in the dissolution cell. However, one limitation is the poor extraction of the free drug, leading to reduced sensitivity (e.g., drug release from nanoparticles depends on the amount of free drug near the particles). A very similar in-vitro perfusion system has been proposed by $\mathrm{Xu}$ and coworkers to measure the release of drugs from solutions; micelles; and small, medium, or large globule size nanoemulsions (15). It is based on tangential flow filtration but uses a different mode of operation. The free drug is removed by controlling the permeate flux. This gives the operator a certain control over the shear forces applied to the dosage form and modulates the drug release (15). The released drug can be removed completely from the donor chamber in less than $10 \mathrm{~min}$. Both techniques, NanoDis and adaptive perfusion, enable a flow-based separation of nanomaterials from the free drug but may need further validation before they can be recommended for broader application.

\section{Method Development}

Method development includes the selection of the membrane pore size based on the particle size of the nanomaterial. The MWCO corresponds to a certain filter pore size but further validation is necessary to confirm a complete separation of the free from the particle-bound fraction.

Quantification of particle retention can be performed using well-defined particle standards. As indicated in the previous sections, most of the commercial fluorescent particle standards release a certain fraction of the label after a few hours. So far, there are no suitable standards with high stability over more than $24 \mathrm{~h}$. In the development of the NanoDis system, particles were loaded with a fluorescent label, and filter retention of the particles was evaluated over $30 \mathrm{~min}$. For longer release assays or release media, the stability of the particle standard with regard to the release of the fluorescent dye must be considered (32). See Table 4 for more information on the parameters to be optimized in the development of flowseparation techniques.

\begin{tabular}{|c|c|}
\hline Flow rate & $\begin{array}{l}\text { In most flow-separation techniques, the flow rate is used to increase the mechanical shear forces applied to the } \\
\text { sample. This leads to a more rapid separation of the sample and a shorter release response. Therefore, the flow rate } \\
\text { plays a key role in the stability of the preparation, and validation should include a quantification of particle stability } \\
\text { under these conditions. }\end{array}$ \\
\hline Release response & $\begin{array}{l}\text { The release response provides a time-resolved measure of the amount of free drug being detected when using } \\
\text { a specific separation process. Commonly, a solution of the free drug is spiked into the donor chamber, and the } \\
\text { amount of the drug that becomes available (the response) is measured in a timeresolved manner. Importantly, the } \\
\text { time between the addition of free (solubilized drug) and the detection in the assay is reported. As compared to a } \\
\text { conventional spiking recovery test, the separation time is not considered negligible. }\end{array}$ \\
\hline Sample treatment and dilution & $\begin{array}{l}\text { The sample treatment including the exact amount of elution liquid used for the separation is required. An effect of the } \\
\text { dilution and elution protocol on the release rate should be excluded. }\end{array}$ \\
\hline Membrane integrity test & $\begin{array}{l}\text { The membrane integrity measures the integrity of the dialysis membrane in response to the separation experiment. } \\
\text { For example, validation of the NanoDis system included a measurement of water permeation. Other measures may } \\
\text { include membrane permeation experiments before and after the release experiment was carried out. }\end{array}$ \\
\hline Particle retention & $\begin{array}{l}\text { Particle retention over time can be measured by using labeled particle standards. It is generally recognized that } \\
\text { there are no particle standards with sufficient stability. The release of the label and the particle integrity may pose a } \\
\text { challenge in the evaluation of particle retention. }\end{array}$ \\
\hline Particle collection & $\begin{array}{l}\text { For time-consuming flow-separation processes, the cross-flow may have an impact on particle stability. Therefore, the } \\
\text { stability of the particle systems should be measured. For example, AF4 enables the quantification of the exact number } \\
\text { of particles collected. For other studies, the in-vitro stability may provide sufficient information. }\end{array}$ \\
\hline Material interaction & $\begin{array}{l}\text { Material interactions play an important role during method development. Membrane adsorption of the drug should } \\
\text { be investigated. In this context, exact hydrodynamics play an important role. It is therefore recommended to carry } \\
\text { out the material interaction studies under conditions very similar to the release test including the setup, medium, } \\
\text { temperature, and other standardized parameters. }\end{array}$ \\
\hline
\end{tabular}




\section{SAMPLE-AND-SEPARATE METHODS}

While the above mentioned techniques assure a continuous detection of the drug released from the nanomaterial, sample-and-separate methods utilize a rapid purification step to extract the analyte. These methods will be discussed in the following section.

\section{Filtration Methods \\ Background}

Dissolution testing commonly utilizes filters to prevent undissolved particles from being unintentionally collected at each time point, resulting in inaccurate results. If undissolved particles are sampled, they can dissolve after collection and before measurement, which creates an artificially faster release profile. During the separation, mechanical pressure is applied to the dosage form. This may have a significant influence on the drug release. Most common methods include syringe filtration $(8,26$, 27) and centrifugal filter methods (33).

Frilled filters typically have larger pore sizes of several microns, which would not be suitable for nanoparticles. For comparison, membrane filters typically cover a size range of 200-1000 $\mathrm{nm}$ and may be suitable depending on the size of the nanoparticle under investigation. However, it is important to remember that the pore size of membrane filters is commonly reported as the largest pore size in the filter, whereas for frilled filters, the pore size is reported as the average pore size in the filter. The development of smaller pore size filters has also been driven by needs in the sterile product field. There are smaller membrane filters of $100-\mathrm{nm}$ pore size that specifically remove mycoplasma, which are the smallest class of free-living microorganisms. These smaller pore size filters may add value for nanoparticle filtration applications. For traditional dissolution testing, frilled or syringe filters are common, however, some separation systems such as the TNO TIM-1 system (TNO Zeist TIM B.V., Netherlands) claims to use filters with a pore size of $50 \mathrm{~nm}$.

In addition, filter techniques normally used to prepare, isolate, or concentrate nanoparticles during nanoparticle synthesis could be applied during product performance testing. Ultrafiltration in the single-digit nanometer particle range is also available and have been evaluated using quantum dots (34).

\section{Method Development}

To select an appropriate filter for use in product performance testing, chemical compatibility, low level of extractables, and low drug substance and excipient binding are the most important considerations. Importantly, not all extractables interfere with the separation process but can sometimes lead to higher variability of the assay.

For nanoparticles, the pore size of the filter needs to be smaller than the nanoparticles being tested, which can be extremely challenging. As a practical starting point, the filter should be a smaller pore size than at least $90 \%$ of the nanoparticles being tested, based on the nanoparticle size distribution. Several approaches have been made to validate the filtration efficiency. For example, particle penetration has been evaluated using nanoparticle tracking analysis (NTA) to identify particles in the filtrate, together with a quantification of the percentage of the drug retained by the filter in a suitable dispersion medium (8). In this context, the dispersion medium should not solubilize significant amounts of the drug (8). However, the small pore size of the filter may raise challenges if the nanoparticles and pores are of similar size; consequently, filter blockage can occur or the diffusion or transit of the nanoparticle through the pore may be sufficiently slow as to result in separation based on time and diffusion rate. It is highly recommended to perform product performance testing with filters of various pore sizes to optimize the choice of filter. Ideally, one filter with a pore size within the smallest $10 \%$ of the nanoparticle distribution size range and one filter with a pore size within the largest $10 \%$ of the nanoparticle distribution size range should be used.

The flow of the media across the filter can also impact the filter performance. Dead-end filtration, where the entire fluid passes through the filter, can be problematic because the nanoparticles can concentrate at the pores and create a boundary layer that can lead to nanoparticle aggregation or filter clogs. Tangential flow filtration can overcome these challenges. As the name implies, this approach provides a flow of media and particles at a tangent to the pores. If pressure is added to the system, the smaller particles are encouraged to penetrate the membrane while the tangential flow prevents the aggregation and boundary layer events that might result in filter clogs.

Other factors that can affect filter performance are the filter size and area, as well as the physicochemical characteristics of the media (e.g., temperature, viscosity, $\mathrm{pH}$, and ionic strength). Some of the most recent methods applied in the filtration of nanoparticles have been reviewed by D'Souza in 2014 (35) and Wacker and Nothnagel in 2018 (27). For example, Weng et al. (36) compared different filtration techniques for nanoparticle formulations with a particle diameter below $100 \mathrm{~nm}$. 
While filtration using filter pore sizes of 450 or $200 \mathrm{~nm}$ was not predictive and led to a strong burst effect, centrifugal ultrafiltration was found to be more reliable (36). Various literature sources have helped identify the criticality of the filtration of nanoparticles. See Table 5 for selected key parameters to study and document the impact.

\section{Solid-Phase Extraction Methods Background}

Solid-phase extraction (SPE) is a popular technique using cartridges filled with sorbents of small particle size for extraction or purification of compounds from liquid samples or solid matrices. A large number of studies have been published for the SPE-based separation of nanoparticle formulations. However, most are for the extraction of active pharmaceutical ingredients from physiological matrices after the nanoparticle formulations are administered. A recent investigation reported effective separation and quantification of the free and the entrapped drugs from lipid nanoparticles (37, 38), setting a starting point for in-vitro drug release and performance testing of drug-loaded lipid nanoparticles formulations.

SPE methods developed for drug-loaded lipid nanoparticles are based on the reverse-phase SPE concept, relying on the van der Waals interactions between the hydrophobic analyte and the hydrophobic sorbent. This leads to stronger retention of the lipophilic analyte, whereas in the hydrophilic mobile phase, the lipid nanoparticles are eluted much more rapidly. As SPE sorbents consist of silica beads with pores smaller than the nanoparticle diameter but larger than the analyte as a molecular entity, the separation is also driven by the significant size difference between both entities.

\section{Method Development}

So far, two commercially available cartridges are reported for separation and quantification of the free and the entrapped drugs from lipid nanoparticles $(37,38)$. Method development generally includes the following steps:

- A medium is introduced into the SPE cartridge to prepare the column for separation.

- The cartridge is conditioned with a separation medium.

- The formulation is loaded into the cartridge.

- The nanoparticles are eluted in the first fraction.

- The free drug is eluted in a second fraction.

All of the steps are conducted using a vacuum chamber with controlled negative pressure. The free fraction is analyzed using a suitable quantification method (e.g., HPLC).

For SPE methods applied to separate free and encapsulated drug in liposome formulations from physiological matrices, in either animal or human plasma or tissues, the dilution and addition of internal standards is required (39-41). More sensitive separation methods and smaller volumes are used. There are several critical parameters in the separation of nanoparticles using SPE. For example, Varache et al. indicated that the nanoparticles could interact with the sorbent materials with a strong effect on the outer layers of

\begin{tabular}{|c|c|}
\hline Volume & $\begin{array}{l}\text { Before filter membranes can be used, filter saturation requires a certain volume of the liquid to be filtered and } \\
\text { discarded. An investigation of this volume is mandated to reduce effects of membrane adsorption on the outcome } \\
\text { of the investigation. Also, particularly for biorelevant methods, the volume of the medium that can be filtered at } \\
\text { acceptable pressure must be determined. }\end{array}$ \\
\hline Hydrodynamics & $\begin{array}{l}\text { Hydrodynamics plays a certain role for many filtration methods. The surface area and the pressure applied to the } \\
\text { filter membrane have a strong impact on the selectivity of the method for the particle population. }\end{array}$ \\
\hline Filter pore size & $\begin{array}{l}\text { The filter pore size influences selectivity of the method for a certain size particle. Commonly, the average pore size } \\
\text { is reported. Therefore, the filter permeation of particles must be evaluated before a certain filter pore size can be } \\
\text { used. Common practices include the filtration of stable particle systems and detection of the material in the filtrate } \\
\text { or a quantification of the particle concentration using particle characterization methods such as dynamic light } \\
\text { scattering and nanoparticle tracking analysis. These methods have a much lower sensitivity and should therefore } \\
\text { be seen as supplementary semiquantitative evidence. }\end{array}$ \\
\hline $\begin{array}{l}\text { Filtration parameters and } \\
\text { observations }\end{array}$ & $\begin{array}{l}\text { For syringe filtration, observations such as the pressure applied to the filter for different media should be } \\
\text { reported. For ultrafiltration, centrifugation speed or pressure, as well as ultrafiltration time and the design of } \\
\text { the ultracentrifugation device, with regard to separation or stability of the nanoparticles (aggregation) should be } \\
\text { reported. }\end{array}$ \\
\hline Filter integrity and performance & $\begin{array}{l}\text { The filter integrity should be confirmed with a bubble point test or a similar method after completion of the } \\
\text { experiment. Also, the influence of the release medium on filter integrity should be carefully considered. For } \\
\text { example, organic solvents or surfactants may have a strong influence on the surface characteristics of the filter } \\
\text { membrane. }\end{array}$ \\
\hline
\end{tabular}


the nanoparticles and colloidal stability (38). This leads to an overestimation of the free drug content and a significant increase in coeluted excipients. For the same type of drug-loaded lipid nanoparticles, these effects were considerably reduced after changing to another column type. Also, this led to a reduction in the coeluted excipients and maintenance of colloidal integrity after separation even at lower nanoparticle concentrations and reduced particle diameter (38). See Table 6 for critical parameters to be investigated, validated, and reported.

\section{Centrifugation Methods Background}

Centrifugation is a mechanical process involving the use of an outward force in a rotating reference frame to separate particles of different sizes and densities. The rate of centrifugation is defined by the angular velocity and is typically expressed in revolutions per minute (rpm). The gravitational force ( $g$-force) equivalent is defined as the acceleration force per unit mass. Ag-force of 7 is equal to the gravitational acceleration on Earth $(9.8 \mathrm{~m} /$ $\mathrm{s}^{2}$ ). Consistent with Stokes' Law, separation in a centrifuge depends on particle size, shape, and density of the medium. Other parameters include the viscosity of the medium and the rotor speed. High-density components of the mixture drift far from the axis of the centrifuge while the less-dense components migrate towards the axis. The denser the component is, the faster it sediments in the centrifugal field. There are two types of ultracentrifuges available, including analytical and preparative systems.
Analytical ultracentrifuges are equipped with optical detection systems that allow following the centrifugation process in real time. Such systems may use ultraviolet (UV) light absorption, refractive index interference (RII), or combinations of these. Analytical ultracentrifugation characterizes the sample during centrifugation and provides information based on sedimentation velocity, viscosity, and concentration. With analytical ultracentrifugation, it is possible to monitor the variations in sample concentration as a function of the applied centrifugal force. The technique is used in sedimentation velocity and sedimentation equilibrium studies, providing the key to the analysis of macromolecules (42). Preparative ultracentrifuges are mostly used to process the samples for further analysis. The most common methods are differential centrifugation sedimentation and density-gradient centrifugation. Centrifugation offers a fine separation of particle populations and widely depends on the sedimentation behavior. Therefore, particle populations broadly distributed in size may not allow a complete separation of the nanomaterial from the release medium. Reference experiments may include a filtration step after centrifugation to make sure that no further particles are present in the supernatant.

\section{Method Development}

Differential centrifugation sedimentation (DCS) is used to separate multiple fractions within a sample. Particles of different densities or sizes will sediment at different rates. This technique measures the time required for

Table 6. Key Parameters to be Explored and Documented for the Evaluation of Release Experiments Using SPE Methods

\begin{tabular}{|c|c|}
\hline $\begin{array}{l}\text { SPE cartridge material or } \\
\text { sorbent }\end{array}$ & $\begin{array}{l}\text { A wide choice of sorbents for conventional SPE exists, including nonpolar, polar, ion exchange, and mixed mode } \\
\text { chemistries. For the SPE of free drug from lipid nanoparticles, the hydrophobic sorbent is preferred. }\end{array}$ \\
\hline SPE pore size and particle size & The two parameters should be correlated with the sizes of the drug molecule and the nanoparticles to be separated. \\
\hline Sorbent amount and volume & $\begin{array}{c}\text { The SPE sorbent amount and volume should be considered when selecting cartridges for a particular sample type and } \\
\text { volume. }\end{array}$ \\
\hline $\begin{array}{l}\text { Medium type, elution protocol, } \\
\text { and volume }\end{array}$ & $\begin{array}{l}\text { The SPE cartridge needs to be cleaned and equilibrated before loading the nanoparticle sample. The cleaning medium } \\
\text { should be the same or very similar to the eluting medium for the free drug elution. The equilibration medium should } \\
\text { be the same or similar to the eluting medium for the nanoparticles. For nanoparticle elution, buffered or unbuffered } \\
\text { aqueous medium can be selected during method development on the basis of the nanoparticles' property. For free } \\
\text { drug elution, organic solvent can be required. }\end{array}$ \\
\hline Elution pressure & $\begin{array}{l}\text { Negative pressure is required to facilitate and accelerate the SPE process. The negative pressure needs to be } \\
\text { controlled for method repeatability and optimized to avoid affecting the stability of the nanoparticles and } \\
\text { overestimating the amount of the fraction released. }\end{array}$ \\
\hline Release response & $\begin{array}{l}\text { The release response provides a time-resolved measure of the amount of free drug being detected when using } \\
\text { a specific separation process. Commonly, a solution of the free drug is spiked into the donor chamber, and the } \\
\text { amount of the drug that becomes available (the response) is measured in a timeresolved manner. Importantly, the } \\
\text { time between the addition of free (solubilized) drug and its detection in the assay is reported. As compared to a } \\
\text { conventional spiking recovery test, the separation time is not considered negligible. }\end{array}$ \\
\hline Sample treatment and dilution & $\begin{array}{l}\text { The sample treatment including the exact amount of elution liquid used for the separation is required. Any effect of } \\
\text { the dilution and elution protocol on the release rate should be excluded. }\end{array}$ \\
\hline
\end{tabular}


particle sedimentation in a fluid when exposed to the centrifugal field. Since very small particles in the nanoscale will sediment very slowly, the sedimentation needs to be greatly accelerated. The centrifuge can cover a rather wide dynamic range of particle sizes from 0.002$80 \mu \mathrm{m}$. DCS has also been used to measure both the size and density of particles by performing two independent experiments using media with different densities (43).

Density gradient centrifugation utilizes media with a density gradient to separate particles. Methods include rate-zonal centrifugation and isopycnic centrifugation. They differ in the way particles are separated across the gradient. Rate-zonal centrifugation uses the differences in hydrodynamic behavior to separate objects. In this technique, a sample solution containing particles to be separated is layered on a preformed linear-gradient column. The sample solution creates a negative gradient at the top of the column thus preventing the sample from premature sedimentation. Under centrifugal forces, the particles will sediment through the gradient column in separate zones, each zone consisting of particles characterized by their size and sedimentation rate. In the past, multiphase systems have been applied as media for rate-zonal centrifugation to separate nanoparticles of different shapes and sizes (44). Reaction products including nanorods, nanospheres, and large particles have been separated in a three-phase system. Approximately $8 \%-99 \%$ of the particles have been separated in less than 10 min using a benchtop centrifuge.

Isopycnic centrifugation improves the quality of nanoparticle separation and relies on a density gradient and ultracentrifugation to separate components according to subtle density differences. The particle separation depends solely on density. In isopycnic separation, particles are mixed with the gradient solution, and during centrifugation, they move until they reach the gradient phase, which equals their density (isopycnic or equilibrium point). Because the density of the gradient medium is always higher than the density of particles, these will never sediment regardless of the centrifugation time. Continuous gradients may be used in isopycnic centrifugation, however, discontinuous gradients in which particles form bands at the interface between the density gradient layers are more suitable for the separation of some biological samples, such as the separation of lymphocytes from whole blood (42).

Since the density of biological particles is sensitive to the osmotic pressure of the gradient, isopycnic separation may vary significantly depending on the gradient medium used. Isopycnic centrifugation can be applied for the purification of large volumes of biomolecules and for the determination of densities of various particles.

Isopycnic density-gradient centrifugation method reaches a limitation when it is extended to the separation of metal nanoparticles. Such a method requires that the components for separation have densities within a gradient range. Aqueous density gradient media usually have densities less than $1.4 \mathrm{~g} / \mathrm{cm}^{-3}$, which is much less than the density of metal nanoparticles. Size or shape separation of such heavy nanocrystals remains an issue, both in their preparation and utility for various applications (45). See Table 7 for more information on the key parameters to be optimized during method development.

\section{OTHER METHODS}

Recent approaches in release testing include novel separation methods such as the use of a phase-separated barrier to detect release and absorption of the drug (Fig. 3). This creates a hydrodynamic boundary that is difficult for the nanoparticle to cross, and the sampling probe can be placed in a specific location that is more likely to collect only dissolved drug. As a result, a more conventional pore size filter could potentially be utilized. Another additional

Table 7. Key Parameters to be Explored and Documented for the Evaluation of Release Experiments Using Centrifugation Methods

\begin{tabular}{|c|c|c|}
\hline Medium composition & $\begin{array}{c}\text { The selection of the medium is commonly based on particle buoyancy density or/and rate of sedimentation, but } \mathrm{pH} \text { and ionic } \\
\text { strength can play a role as well. The medium should minimize interference with the assay procedures or reaction potential } \\
\text { with tubes. Additionally, hyperosmotic or hypoosmotic media should be avoided if the particles are osmotically sensitive. } \\
\text { The centrifugation step is considered a separation step and, as such, should have minor impact on the release behavior. }\end{array}$ \\
\hline Centrifugal temperature & $\begin{array}{c}\text { Considering centrifugation as a separation protocol which should not affect the release rate, the centrifugal temperature can } \\
\text { be used to slow down the release process. It should be monitored, and the effect on the release rate should be measured. }\end{array}$ \\
\hline Centrifugal speed & $\begin{array}{c}\text { The centrifugal speed can be used to shorten the separation time. To assure reliability of the assay, the release response and } \\
\text { potential for release during the separation step should be considered. }\end{array}$ \\
\hline Release response & $\begin{array}{c}\text { The release response expresses the amount of free drug being detected when using a specific separation process. } \\
\text { Commonly, free drug is added during the assay, and the time and amount of the drug that becomes available (the response) } \\
\text { is measured. Importantly, the time between the addition of the free drug and its detection in the assay is reported. The } \\
\text { separation time is not considered negligible. }\end{array}$ \\
\hline
\end{tabular}


boundary for the nanoparticle to cross might be created through the use of a two-phase media. If an appropriate second liquid phase can be identified that dissolved drug can enter but insoluble particles cannot, due to the properties of the liquid-liquid interface, this could be an interesting opportunity with current filters.

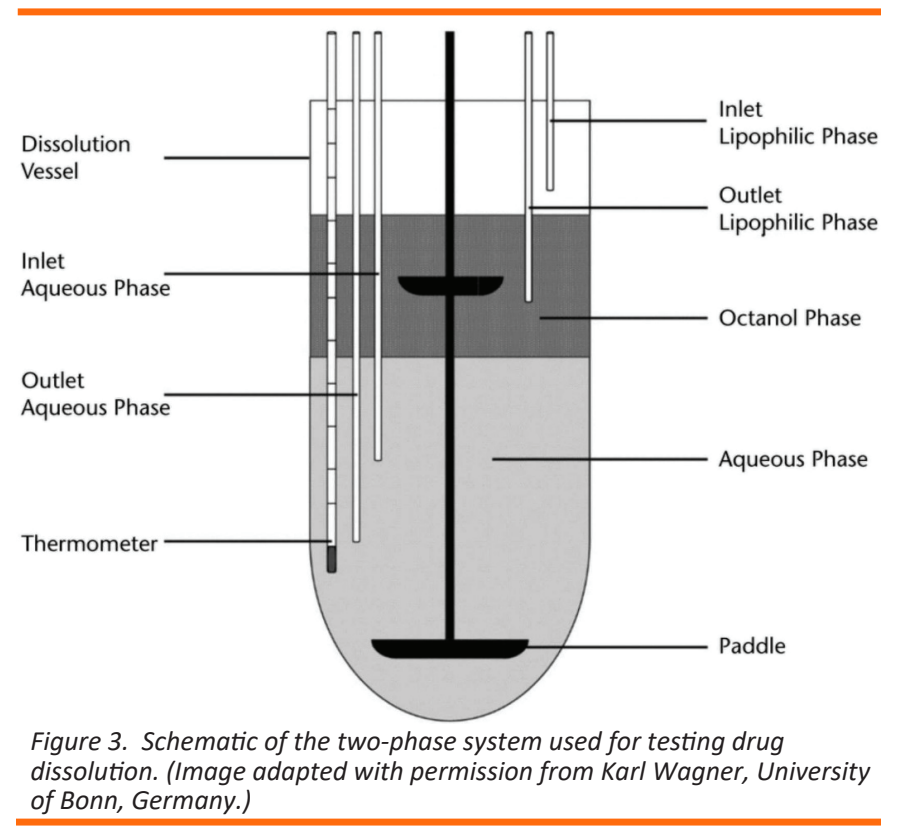

This opportunity is magnified by the increase in the usage and the expertise needed to design custom ionic liquids for pharmaceutical use. Ionic liquids have been shown to be highly valuable in separating components in aqueous two-phase systems (46).

Another example of use for such a nonconventional barrier for separation is the application of hydrogel assays to simulate the subcutaneous route of administration. Other than the two-phase release assay, this model is not based on a compendial instrument. The release is measured by detection of the drug movement using fluorescence imaging or sampling from the release environment. In the past, different types of hydrogels have been used for this application. It does not qualify for a complete separation of the free drug from the nanoparticles. More importantly, it provides a simple measure for formulation mobility in a complex three-dimensional network that can be correlated to particle mobility in specific tissues $(47,48)$.

\section{GENERAL RECOMMENDATIONS FOR} VALIDATION

Validation of release assays applied to separate nanomaterials from the free drug should generally include the selectivity (separation) of the method for the fraction of interest and the sensitivity of the assay for small differences in the drug release behavior (discrimination). Both require a different set of experiments that depend on the exact method under investigation.

\section{Particle Selectivity}

To validate the selectivity of the particle extraction method for the fraction of interest, the separation method should be performed with a valid particle standard. This may be more challenging for long-term experiments in biorelevant fluids, where common particle standards (e.g., fluorescently labeled beads, gold nanoparticles) do not have sufficient stability or may dissolve over time. The detection of the carrier material or a suitable and stable label are the preferred option, but particle counting and qualitative evidence (e.g., using particle size measurement) can also be used. For example, Jung et al. describe the quantification of the particle concentration before and after filtration of the nanocrystals under investigation using nanoparticle tracking analysis (8). The reference experiments also included quantification of the free drug after filtration (8). In another study, the evaluation of particle accumulation in the different layers of the filter membrane was described (26).

\section{Mechanism of Release}

Currently, nanomaterial-based drug products include a wide variety of dosage forms. They have been summarized in USP Chapter <1153>. In every performance test, the assay reflects some aspects of the physiological microenvironment that are responsible for the in-vivo performance. Therefore, the administration route and exact hydrodynamics play an important role. To effectively simulate the mechanisms relevant to discriminate their performances, the shear forces applied to the dosage form must be precisely controlled. This effect is particularly important for nanomaterials because the stability of colloids is more difficult to determine as compared to other dosage forms.

For example, a reduction in the integrity of the lipid bilayer of liposomes can be responsible for a much faster drug release. For the parenteral route of administration, the mechanism of release may differ between different injection sites and cannot be explained using our knowledge from the peroral route of administration. Therefore, particularly for those nanomaterials applied in non-peroral dosage forms, a thorough investigation of the exact mechanism of release is required. Aspects to be considered for apparatus and release medium selection for parenteral product performance will be discussed in more detail in a further stimuli article focusing on 
injections and implanted drug products. For inhalation products, a similar stimuli article is currently prepared.

\section{Release Response}

With a growing number of nonconventional assays being utilized to evaluate the drug release, the time required for the separation of the drug from the release medium becomes a critical aspect in the experimental design. Most conventional separation methods such as syringe filtration or centrifugation $(8,26,49)$ assure a purification of the free drug from their matrices within a short period of less than a minute and without further dilution of the sample. Some more recent techniques require several preparation steps and separation times ranging from a few minutes to hours. For example, the separation of nanopharmaceuticals from the free drug, proteins, and protein-drug complexes by $\operatorname{AF} 4$ requires $(28,29)$ separation times of $40-60 \mathrm{~min}(28,29)$.

Therefore, validation of the assay should include a measurement of the response to a particular amount of drug set free in the assay. This can, for example, be achieved by the injection of a drug solution during the dissolution test. The time between injection and release response should be reported. Importantly, this includes the absolute time between sample collection and detection of the fraction including the separation time. The release response is a time-resolved evaluation of the "spiking-recovery", integrating the elements of the kinetic measurement in the validation protocol.

\section{Sample Treatment and Changes in the Microenvironment}

While the established filtration and dialysis methods carry out a separation of the carrier from the free drug in the release medium, some assay systems require dilution of the sample. For example, flow-separation methods and SPE require an elution medium that may differ from the release medium under investigation. Under these conditions, the influence of the elution medium on the sample and on the drug release in particular should be investigated. This can be achieved by using an orthogonal method or by using appropriate references. Different dilution protocols and concentrations should be tested to see differences in the release behavior. This may, for example, include the extraction of the free drug at different pressures in the SPE setup.

\section{CONCLUSION}

With a wide variety of nanomaterial-based drug products entering the US market and the unique challenges they present to conventional drug release testing methods, there is a need for guidance on testing their in-vitro product performance. As presented in this article, a dedicated USP chapter $(<1153>)$ now enumerates the different types of nanomaterial and outlines the suggested testing to be performed. Additional guidance for some of these products is currently included in USP chapters relating to the dosage delivery format. However, we have identified a gap in coverage of more specific guidance on selection of appropriate testing methodology, method developments, and validation of release assays. In this work, we have highlighted some of the more common testing method options, including suggested critical method parameters for each to specify and validate. Also, we have included a brief section of general recommendations for validation of a product performance testing method for various nanomaterialbased drug products. In addition to considerations related to the particle size and size distribution of the dosage form, the medium composition must be taken into account during method development, and the release mechanism must be elucidated. This investigation should link the measured parameters (e.g., release, degradation) to the expected performance and provides an important cornerstone of method development. Finally, the New Advancements in Product Performance Testing Expert Panel opens this Stimuli article for public consultation and feedback. We would like to encourage our readers to provide their valuable comments and hope to discuss the outcomes of this process in the near future.

\section{ACKNOWLEDGEMENTS}

This manuscript was peer-reviewed by the following reviewers. The authors thank them for their valuable contributions to this work:

Deirdre D'Arcy, Trinity College, Dublin, Ireland; Zhigang Sun, Luye Pharma Group Ltd., Princeton, New Jersey, USA; Helmut Rockstroh, F. Hoffmann-La Roche Ltd., Staufen, Germany; Lawrence Block, Duquesne University, Carnegie, Pennsylvania, USA; Richard Moreton, FinnBrit Consulting, Waltham, Massachusetts, USA; Salish Singh, Moderna, Cambridge, Massachusetts, USA; Yongchao Su, Merck \& Co., Chalfont, Pennsylvania, USA; Kevin Swiss, Vitruvias Therapeutics LLC, Blaine, Washington, USA; Xiaoming Xu, Division of Product Quality Research Office of Testing and Research, Office of Pharmaceutical Quality, Center for Drug Evaluation and Research, US Food and Drug Administration, Maryland, USA; Thomas Tice, Evonik Corporation, Indian Springs Village, Alabama, USA; John P. Hammond, Starna Scientific, Romford, UK; Anthony Hickey, RTI International, Chapel Hill, North Carolina, USA; June Liang, Office of Foods and Veterinary 
Medicine, Center for Veterinary Medicine, US Food and Drug Administration, Maryland, USA.

The authors also acknowledge Bjorn Fahler (Pharma Test Apparatebau AG), Emre TOreli (MyBiotech GmbH), and Karl Wagner (University of Bonn) for providing information and material on the release methods under development.

\section{REFERENCES}

1. Marques MRC, Choo Q, Ashtikar M, Rocha TC, BremerHoffmann S, Wacker MG. Nanomedicines - Tiny particles and big challenges. Adv Drug Deliv Rev. 2019;151-152:23-43.

2. Mitchell MJ, Billingsley MM, Haley RM, Wechsler ME, Peppas NA, Langer R. Engineering precision nanoparticles for drug delivery. Nature Rev Drug Disc. 2020.

3. Wallenwein CM, Nova MV, Janas C, Jablonka L, Gao GF, Thurn M, Albrecht $\mathrm{V}$, Wiehe A, Wacker MG. A dialysis-based in vitro drug release assay to study dynamics of the drug-protein transfer of temoporfin liposomes. Eur J Pharm Biopharm. 2019;143:44-50.

4. Olenick LL, Troiano JM, Vartanian A, Melby ES, Mensch AC, Zhang L, Hong J, Mesele 0, Qiu T, Bozich J, Lohse S, Zhang X, Kuech TR, Millevolte A, Gunsolus I, McGeachy AC, Dogangun M, Li T, Hu D, Walter SR, Mohaimani A, Schmoldt A, Torelli MD, Hurley KR, Dalluge J, Chong G, Feng ZV, Haynes CL, Hamers RJ, Pedersen JA, Cui Q, Hernandez R, Klaper R, Orr G, Murphy CJ, Geiger FM. Lipid corona formation from nanoparticle interactions with bilayers. Chem. 2018;4:2709-2723.

5. Gao GF, Ashtikar M, Kojima R, Yoshida T, Kaihara M, Tajiri T, Shanehsazzadeh S, Modh H, Wacker MG. Predicting drug release and degradation kinetics of long acting microsphere formulations of tacrolimus for subcutaneous injection. J Control Release. 2021;329:372-384.

6. Jose J, Charyulu RN. Prolonged drug delivery system of an antifungal drug by association with polyamidoamine dendrimers. Int J Pharm Invest. 2016;6:123-127.

7. Jablonka L, Ashtikar M, Gao G, Jung F, Thurn M, Preuss A, Scheglmann D, Albrecht V, Roder B, Wacker MG. Advanced in silica modeling explains pharmacokinetics and biodistribution of temoporf1n nanocrystals in humans. I Control Release. 2019;308:57-70.

8. Jung F, Thurn M, Krollik K, Gao GF, Hering I, Eilebrecht E, Emara Y, Weiler M, GOnday-TUreli N, TUreli E, Parnham MJ, Wacker MG. Predicting the environmental emissions arising from conventional and nanotechnology-related pharmaceutical drug products. Environ Res. 2021;192:10219.

9. Jablonka L, Ashtikar M, Gao GF, Thurn M, Modh H, Wang JW, Preuss A, Scheglmann D, Albrecht V, Roder B, Wacker MG. Predicting human pharmacokinetics of liposomal temoporfin using a hybrid in silica model. Eur J Pharm Biopharm. 2020;749:121-134.

10. Tang J, Srinivasan S, Yuan W, Ming R, Liu Y, Dai Z, Noble CO, Hayes ME, Zheng N, Jiang W, Szoka FC, Schwendeman A. Development of a flow-through USP 4 apparatus drug release assay for the evaluation of amphotericin B liposome. Eur J Pharm Biopharm. 2019;134:107-116.

11. Bhardwaj U, Burgess DJ. A novel USP apparatus 4 based release testing method for dispersed systems. Int J Pharm. 2010;388:287-294.

12. Janas C, Mostaphaoui Z, Schmiederer L, Bauer J, Wacker MG. Novel polymeric micelles for drug delivery: material characterization and formulation screening. Int J Pharm. 2016;509:197-207.

13. Meng $M$, Gao J, Wu C, Zhou X, Zang X, Lin X, Liu H, Wang C, Su $H$, Liu K, Wang Y, Xue $X$, Wu J. Doxorubicin nanobubble for combining ultrasonography and targeted chemotherapy of rabbit with VX2 liver tumor. Tumor Biol. 2016;37:8673-8680.

14. Ali $\mathrm{HH}$, Hussein AA. Oral nanoemulsions of candesartan cilexetil: formulation, characterization and in vitro drug release studies. AAPS Open. 2017;3:4.

15. D. Patel, Y. Zhang, Y. Dong, H. Qu, D. Kozak, M. Ashraf, X. Xu. Adaptive perfusion: an in vitro release test (IVRT) for complex drug products. J Control Release. 2021;333:65-75.

16. Fugit KD, Anderson BD. Dynamic, nonsink method for the simultaneous determination of drug permeability and binding coefficients in liposomes. Mol Pharm. 2014;11:1314-1325.

17. Kovshova T, Osipova N, Alekseeva A, Malinovskaya J, Belov A, Budko A, Pavlova G, Maksimenko 0, Nagpal S, Braner S, Modh H, Balabanyan V, Wacker MG, Gelperina S. Exploring the interplay between drug release and targeting of lipid-like polymer nanoparticles loaded with doxorubicin. Molecules. 2021;26.

18. Petersen S, Fahr A, Bunjes H. Flow cytometry as a new approach to investigate drug transfer between lipid particles. Mol Pharm. 2010;7:350-363.

19. $\mathrm{Xu} X, \mathrm{Khan} M A$, Burgess DJ. A two-stage reverse dialysis in vitro dissolution testing method for passive targeted liposomes. Int J Pharm. 2012;426:211-218.

20. Gao Z, Westenberger B. Dissolution testing of acetaminophen suspension using dialysis adapter in flow-through apparatus: a technical note. AAPS PharmSciTech. 2012;13:944-948.

21. Janas C, Mast MP, Kirsamer L, Angioni C, Gao F, Mantele W, Dressman J, Wacker MG. The dispersion releaser technology is an effective method for testing drug release from nanosized drug carriers. Eur J Pharm Biopharm. 2017;115:73-83.

22. Zambito Y, Pedreschi E, Di Colo G. Is dialysis a reliable method for studying drug release from nanoparticulate systems?-A case study. Int J Pharm. 2012;434:28-34.

23. Protein clean-up technical handbook. Thermo Fisher Scientific; 2016.

24. Duke SD. Technical Note (TN) - 020.03: Particle retention testing of 0.05 to 0.5 micrometer membrane filters. Thermo Fisher Scientific; 2003.

25. Xie L, Beyer S, Vogel V, Wacker MG, Mantele W. Assessing the drug release from nanoparticles: overcoming the shortcomings of dialysis by using novel optical techniques and a mathematical model. Int J Pharm. 2015;488:108-119.

Dissolution 
26. Jung F, Nothnagel L, Gao F, Thurn M, Vogel V, Wacker MG. A comparison of two biorelevant in vitro drug release methods for nanotherapeutics based on advanced physiologicallybased pharmacokinetic modelling. Eur J Pharm Biopharm. 2018;127:462-470.

27. Nothnagel L, Wacker MG. How to measure release from nanosized carriers? Eur J Pharm Sci. 2018;120:199-211.

28. Caputo F, Mehn D, Clogston JD, Rosslein M, Prina-Mello A, Borgos SE, Gioria S, Calzolai L. Asymmetric-flow field-flow fractionation for measuring particle size, drug loading and (in) stability of nanopharmaceuticals. The joint view of European Union Nanomedicine Characterization Laboratory and National Cancer Institute - Nanotechnology Characterization Laboratory. J Chromatography A. 2021;1635:461767.

29. Holzschuh S, Kaess K, Fahr A, Decker C. Quantitative in vitro assessment of liposome stability and drug transfer employing asymmetrical flow field-flow fractionation (AF4). Pharm Res. 2016;33:842-855.

30. Parat J, Caputo F, Mehn D, Hackley VA, Calzolai L. Physical characterization of liposomal drug formulations using multidetector asymmetrical-flow field flow fractionation. J Control Rel. 2020;320:495-510.

31. Quattrini F, Berrecoso G, Crecente-Campo J, Alonso MJ. Asymmetric flow field-flow fractionation as a multifunctional technique for the characterization of polymeric nanocarriers. Drug Del Transl Res. 2021;11:373-395.

32. Snipstad S, Hak S, Baghirov $H$, Sulheim E, M(IIrch Y, Lelu S, van Haartman E, Back M, Nilsson KPR, Klymchenko AS, de Lange Davies $C$, Aslund AKO. Labeling nanoparticles: dye leakage and altered cellular uptake. Cytometry A, 2017;91:760-766.

33. Cipolla D, Wu H, Eastman S, Redelmeier T, Gonda I, Chan HK. Development and characterization of an in vitro release assay for liposomal ciprofloxacin for inhalation. J Pharm Sci. 2014;103:314-327.

34. Chen S.-C, Segets D, Ling T.-Y, Peukert W, Pui DYH. An experimental study of ultrafiltration for sub-10nm quantum dots and sub$150 \mathrm{~nm}$ nanoparticles through PTFE membrane and Nuclepore filters. JMembrane Sci. 2016;497:153-161.

35. D'Souza S. A review of in vitro drug release test methods for nano-sized dosage forms. Adv Pharm. 2014:304757.

36. Weng J, Tong HHY, Chow SF. In vitro release study of the polymeric drug nanoparticles: development and validation of a novel method. Pharmaceutics. 2020;12:732.

37. Guillot A, Couffin A.-C, Sejean X, Navarro F, Umberger M, Lehr C.-M. Solid phase extraction as an innovative separation method for measuring free and entrapped drug in lipid nanoparticles. Pharmaceutical Res. 2015;32.
38. Varache M, Ciancone M, Couffin AC. Optimization of a solidphase extraction procedure for the analysis of drug-loaded lipid nanoparticles and its application to the determination of leakage and release profiles. J Pharm Sci. 2020;109:2527-2535.

39. Song W, Tweed JA, Visswanathan R, Saunders JP, Gu Z, Holliman $\mathrm{CL}$. Bioanalysis of targeted nanoparticles in monkey plasma via LC-MS/MS. Anal Chem. 2019;91:13874-13882.

40. C. Su, H. Yang, H. Sun, J.P. Fawcett, D. Sun, J. Gu. Bioanalysis of free and liposomal amphotericin Bin rat plasma using solid phase extraction and protein precipitation followed by LC-MS/ MS. J Pharm Biomed Anal. 2018;158:288-293.

41. Xie $Y$, Shao $N$, Jin $Y$, Zhang L, Jiang $H$, Xiong $N$, Su F, Xu H. Determination of non-liposomal and liposomal doxorubicin in plasma by LC-MS/MS coupled with an effective solid phase extraction: in comparison with ultrafiltration technique and application to a pharmacokinetic study. J Chromatogr B Analyt Technol Biomed Life Sci. 2018;1072:149-160.

42. Ohlendieck K, Harding SE. Centrifugation and ultracentrifugation, in: A. Hofmann, S. Clokie (Eds.) Wilson and Walker's Principles and Techniques of Biochemistry and Molecular Biology. Cambridge University Press, Cambridge. 2018:424-453.

43. Nontapot K, Rastogi V, Fagan JA, Reipa V. Size and density measurement of core-shell $\mathrm{Si}$ nanoparticles by analytical ultracentrifugation. Nanotechnology. 2013;24:155701.

44. Akbulut 0 , Mace CR, Martinez RV, Kumar AA, Nie Z, Patton $M R$, Whitesides GM. Separation of nanoparticles in aqueous multiphase systems through centrifugation. Nano Letters. 2012;12:4060-4064.

45. Sun X, Tabakman SM, Seo W.-S, Zhang L, Zhang G, Sherlock S, Bai L, Dai H. Separation of nanoparticles in a density gradient: FeCo@C and gold nanocrystals [in English]. Angewandte Chemie 2009;48:939-942.

46. McQueen L, Lai D. Ionic liquid aqueous two-phase systems from a pharmaceutical perspective. Front Chem. 2019;7:135.

47. Beyer S, Xie L, Schmidt M, de Bruin N, Ashtikar M, Ruschenbaum S, Lange CM, Vogel V, Mantele W, Parnham MJ, Wacker MG. Optimizing novel implant formulations for the prolonged release of biopharmaceuticals using in vitro and in vivo imaging techniques. J Control Release. 2016;235:352-364.

48. He S, Jacobsen J, Nielsen CU, Genina N, Ostergaard J, Mu $\mathrm{H}$. Exploration of in vitro drug release testing methods for saquinavir microenvironmental $\mathrm{pH}$ modifying buccal films. Eur J Pharm Sci. 2021;163:105867.

49. Nothnagel L, Jung $F$, Rossmanith $T$, Thurn $M$, Ashtikar $M$, Geisslinger G, Parnham MJ, Wacker MG. Predictive PBPK modeling as a tool in the formulation of the drug candidate TMP001. Eur J Pharm Biopharm. 2019;134:144-152. 